Dom. Cien., ISSN: 2477-8818

Vol. 5, núm.1., ene, 2019, pp. 678-694

\title{
La hipotermia en pacientes sometidos a cirugía abdominal
}

\section{Hypothermia in patients undergoing abdominal surgery}

\section{Hipotermia em pacientes submetidos a cirurgia abdominal}

Marco A. Guerrero-Casagualpa ${ }^{\text {I }}$ marco88_08@hotmail.com

Zuleira V. Torres-Jaramillo II zulev_2289@yahoo.com

Olmedo X. Ruiz-Lara ${ }^{\text {III }}$ olxarula@gmail.com

Andrés S. Rueda-Ruiz ${ }^{\text {IV }}$ andres_rr17@hotmail.com

Edwin D. Betancourth-Justicia V darkedwin77@hotmail.com

Paola F. Mena-Alencastro VI fher2814@gmail.com

Recibido: 27 de noviembre de 2018 * Corregido: 18 de diciembre de 2018 * Aceptado: 20 de diciembre de 2018

I. Médico General Asistencial; Hospital Napoleón Dávila Córdova, Chone, Ecuador.

II. Médico Residente Neonatología; Hospital General, Ibarra, Ecuador.

III. Médico Residente Traumatología; Hospital General, Ibarra, Ecuador.

IV. Médico Residente; Hospital General; Ambato; Ecuador.

v. Médico Residente Ginecología; Obstetricia Hospital General, Ibarra, Ecuador.

VI. Médico General; Emergencia y Centro Obstetrico; Centro de Salud Chimbacalle; Ministerio de Salud Pública; Quito; Ecuador. 


\title{
Resumen
}

Los pacientes que requieren intervenciones quirúrgicas son unos de los más susceptibles de recibir asistencia intensiva, a pesar del notable desarrollo alcanzado por la anestesiología en los procedimientos y agentes anestésicos, así como de las técnicas de reanimación avanzadas y los progresos en la atención al paciente grave, la mortalidad en el grupo de pacientes quirúrgicos es elevada, lo cual repercute en el aumento de la mortalidad en las UCI. La anestesia y la cirugía causan un significativo impacto sobre el balance térmico corporal. El deterioro de la termorregulación a nivel central y la pérdida de calor durante la cirugía determinan hipotermia en un gran porcentaje de pacientes durante la cirugía y luego de ella. Una correcta estrategia de manejo térmico puede reducir las complicaciones y mejorar los resultados en los pacientes quirúrgicos reduciendo así los costos sanitarios. La presente investigación pretende exponer una revisión bibliográfica acerca de la Hipotermia en los pacientes sometidos a cirugía abdominal pasando por las definiciones y actualizaciones en la ciencia médica respecto a ella. El aporte será precisamente exponer la bibliografía encontrada en el tema de investigación propuesto y brindar al lector un orden de ideas sistemático y si se quiere actualizado, sobretodo de artículos científicos disponibles a través de las publicaciones electrónicas de revistas científicas de temas médicos. La literatura coincide que el método más efectivo para contrarrestar la hipotermia es mantener el calentamiento corporal antes de comenzar la cirugía. En el Ecuador existen pocos estudios que muestren la incidencia de ésta afección de manera general o especifica respecto a la cirugía, edad o patología por tanto los datos estadísticos disponibles no proporcionan una referencia importante como para proyectar y analizar un comportamiento ya sea por el manejo de protocolos médicos, o por alguna predisposición poblacional.

Palabras claves: Hipotermia; Anestesia; Cirugía; Abdomen Abierto; Temperatura Corporal.

\begin{abstract}
Patients who need surgical interventions are those who adapt to intensive care, despite the remarkable results, development, anesthesiology and anesthetic agents, as well as advanced resuscitation techniques and those appropriate in patient care. Mortality in the group of surgical patients is higher, which has an impact on the increase in mortality in the ICU. Anesthesia and surgery cause a significant impact on body thermal equilibrium. The deterioration of

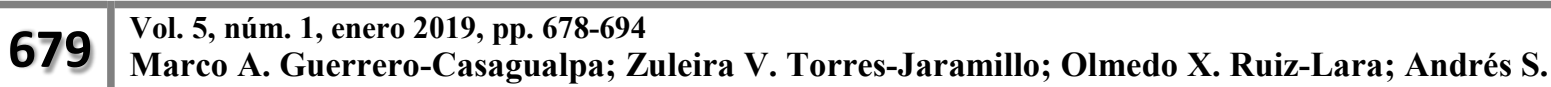


thermoregulation at the central level and the loss of calories during the decisive surgery in a large percentage of patients during and after surgery. A correct thermal management strategy can reduce complications and improve results in surgical patients. The present investigation intends to expose a bibliographical revision about the Hypothermia in the patients submitted to an abdominal surgery. The information was made on the subject of the investigation and the reader was offered. The information was made in a systematic way. The literature coincides with the most effective method to counteract hypothermia. In Ecuador there are few studies that show the incidence of this condition in the general way of care, general information and pathology. Medical protocols, or by some population predisposition.

Key words: Hypothermia; Anesthesia; Surgery; Open Abdomen; Body Temperature.

\section{Resumo}

OMS Os pacientes necessitam de intervenções cirúrgicas são aqueles que se adaptem aos cuidados intensivos, apesar dos resultados notáveis, desenvolvimento, anestesiologia e agentes anestésicos, bem como técnicas de reanimação avançadas e na assistência ao paciente aqueles apropriados. A mortalidade no grupo de pacientes é maior, o que impacta no aumento da mortalidade na UTI. Anestesia e cirurgia causam um impacto significativo no equilíbrio térmico do corpo. A deterioração da termorregulação no nível central e a perda de calorias durante a cirurgia decisiva em uma grande porcentagem de pacientes durante e após a cirurgia. Uma estratégia correta de gerenciamento térmico pode reduzir e melhorar os resultados em pacientes. A presente investigação pretende expor à revisão bibliográfica sobre a hipotermia nos pacientes submetidos à cirurgia abdominal. A informação foi feita sobre o assunto da investigação e o leitor foi oferecido. A informação foi feita de forma sistemática. A literatura coincide com o método mais eficaz para combater a hipotermia. No Equador, existem poucos estudos que mostram a incidência desta condição no modo geral de atendimento, informação geral e patologia. Protocolos médicos, ou por alguma predisposição populacional.

Palavras chave: Hipotermia; Anestesia; Cirurgia; Abdômen Aberto; Temperatura Corporal. 


\section{Introducción.}

Los pacientes que requieren intervenciones quirúrgicas son unos de los más susceptibles de recibir asistencia intensiva, pues muchos presentan procesos fisiopatológicos complejos que deben ser evaluados y atendidos con métodos que solamente pueden desarrollarse por completo en las UCI. Sin embargo, a pesar del notable desarrollo alcanzado por la anestesiología en los procedimientos y agentes anestésicos, así como de las técnicas de reanimación avanzadas y los progresos en la atención al paciente grave, la mortalidad en el grupo de pacientes quirúrgicos es elevada, lo cual repercute en el aumento de la mortalidad en las UCI. (Hernandez, Vinent, Delgado, \& Castillo, 2014)

La anestesia y la cirugía causan un significativo impacto sobre el balance térmico corporal. El deterioro de la termorregulación a nivel central (producido por la anestesia) y la pérdida de calor durante la cirugía (exposición a un ambiente con bajas temperaturas en la sala de operaciones, administración de fluidos) determinan hipotermia en un gran porcentaje de pacientes durante la cirugía y luego de ella. Una correcta estrategia de manejo térmico puede reducir las complicaciones y mejorar los resultados en los pacientes quirúrgicos reduciendo así los costos sanitarios. (Baptista, Rando, \& Zunini, 2010)

La presente investigación pretende exponer una revisión bibliográfica acerca de la Hipotermia en los pacientes sometidos a cirugía abdominal pasando por las definiciones y actualizaciones en la ciencia médica respecto a ella.

\section{Metodología.}

El artículo de revisión es considerado como un estudio detallado, selectivo y crítico que integra la información esencial en una perspectiva unitaria y de conjunto (Icart y Canela, 1994). Un artículo de revisión no es una publicación original y su finalidad es examinar la bibliografía publicada y situarla en cierta perspectiva (Ramos et al, 2003). La revisión se puede reconocer como un estudio en sí mismo, en el cual el revisor tiene un interrogante, recoge datos (en la forma de artículos previos), los analiza y extrae una conclusión. La diferencia fundamental entre una revisión y un trabajo original o estudio primario, es la unidad de análisis, no los principios científicos que se aplican (Day, 2005). 
Según Icart y Canela (1994) la utilidad del artículo de revisión es múltiple. El objetivo fundamental del artículo de revisión intenta identificar qué se conoce del tema, qué se ha investigado y qué aspectos permanecen desconocidos. (Guirao, Olmedo, Angela, \& Esperanza, 2008)

Lo antes expuesto nos permite sustentar el propósito metodológico que acompaña esta revisión bibliográfica cuyo aporte será precisamente exponer la bibliografía encontrada en el tema de investigación propuesto y brindar al lector un orden de ideas sistemático y si se quiere actualizado, sobretodo de artículos científicos disponibles a través de las publicaciones electrónicas de revistas científicas de temas médicos.

\section{Resultados.}

Es propicio comenzar los resultados de esta investigación exponiendo la información relacionada a la cirugía abdominal, por tanto hemos conseguido una publicación de Tavares y colaboradores (2008) sobre Abdomen Abierto.

A pesar de la cada vez más frecuente utilización del cierre temporal de la pared abdominal, no existe una definición operativa de abdomen abierto ni guías universalmente aceptadas que indiquen su realización. El abdomen abierto no es una estrategia quirúrgica inocua; sus complicaciones van desde disturbios hidroelectrolíticos (secundarios a pérdida no estimable de líquidos), formación de fístulas entéricas, abscesos intraabdominales y desarrollo de gigantescos defectos musculoaponeuróticos abdominales, con consecuencias estéticas y funcionales importantes. (Tavares, Andrade, Goné, \& Sánchez, 2008)

El abdomen abierto puede ser el resultado de una estrategia quirúrgica para facilitar una reoperación prevenir o tratar el síndrome compartamental abdominal o ser el resultado de una complicación quirúrgica: dehiscencia de la pared abdominal (por infección o necrosis) o secundario a una catastrófica pérdida de la integridad del abdomen por trauma o tumor. En la actualidad se aconseja utilizar esta técnica de forma planeada en las siguientes circunstancias:

- Cirugía de control de daños. Sepsis intraabdominal severa.

- Síndrome compartamental abdominal. 
- Cuando el cierre primario de la pared abdominal se realiza bajo mucha tensión. (Tavares, Andrade, Goné, \& Sánchez, 2008)

La cirugía de control de daños es uno de los temas más polémicos en la actual cirugía del trauma. El término "cirugía de control de daños" no se aplica solamente al abdomen, sino a otras regiones corporales. En esencia, este principio se refiere a abreviar la manipulación y el tiempo quirúrgicos, practicando las maniobras estrictamente necesarias encaminadas a controlar de manera inmediata las lesiones que ponen en peligro la vida y a apoyar a la restauración de la fisiología del paciente (García, y otros, 2005)

\section{Consideraciones para cirugía de control de daños}

- Trauma abdominal penetrante con presión arterial sistólica menor de $90 \mathrm{mmHg}$.

- Lesiones por proyectil de arma de fuego de alta velocidad o trauma por aplastamiento.

- Trauma multisistémico con trauma abdominal grave.

- Fractura pélvica compleja con lesión intraabdominal asociada.

- Múltiples víctimas que requieren tratamiento quirúrgico y extrema limitación de recursos.

- Trauma militar grave.

Fuente: (García, y otros, 2005)

\section{Parámetros clave usados en la decisión del establecimiento de la cirugía de control de} daños.

- Hipotensión con presión arterial sistólica menor de $90 \mathrm{mmHg}$.

- Hipotermia $<34^{\circ} \mathrm{C}$.

- Coagulopatía APPT > $60 \mathrm{seg}$.

- Acidosis $\mathrm{pH}<7.2$ o déficit de base $>8$.

- Lesión vascular abdominal grave.

- Necesidad asociada del manejo de lesiones extraabdominales que ponen en peligro la vida.

Fuente: (García, y otros, 2005)

\section{Errores comunes en la toma de decisión de la cirugía de control de daños.}

- Retardo en el reconocimiento de la necesidad de la cirugía de control de daños.

- Falla para tomar la decisión en el Departamento de Urgencias. 
- Pobre comunicación con el anestesiólogo, enfermeras y resto del equipo de trauma.

- Monitorización inadecuada de la temperatura del paciente.

- Toma inadecuada de gases arteriales en el Departamento de Urgencias o en el quirófano.

- Inadecuado cálculo de la reposición de volumen.

- Ego quirúrgico.

- Realización de investigaciones innecesarias inmediatamente después del procedimiento de control de daños.

- Rápida transferencia del paciente del quirófano a la Unidad de Cuidados Intensivos.

Fuente: (García, y otros, 2005)

De la investigación se desprende un término interesante para el objeto de estudio que tiene que ver con la "Triada de la muerte" o "Triada Mortal"

Se trata de tres complicaciones la acidosis, la hipotermia y la coagulopatía, que muchas veces se escapan del control médico y se han denominad "Tríada Mortal” por sus nefastas consecuencias; actúan como un círculo vicioso retroalimentándose positivamente cuando no son previstas o prevenidas adecuadamente, y obligan a postergar el tratamiento quirúrgico definitivo con el objetivo de lograr una mayor supervivencia mediante pasos consecutivos y ordenados, basados en la tolerancia fisiológica del paciente. Estas estrategias han sido denominadas "Cirugía de Control de Daños" universalmente concebidas en tres etapas. La primera etapa de control de daño, la hemorragia y la contaminación. La segunda etapa es caracterizada por la corrección de anormalidades físiológicas en una unidad de cuidados intensivos. Los pacientes son calentados y resucitados, y se corrigen los defectos de coagulación. En la fase final de control de daño, el tratamiento se completa en un paciente estable realizando la reparación definitiva de las lesiones. (Gómez, Ramos, Llanes, \& Gonzalez, 2007)

\section{Balance térmico perioperatorio}

Una definición fisiológica de hipotermia es el descenso de la temperatura central mayor a un desvío estándar por debajo de la media, en condiciones basales y en un entorno térmico neutro. En el hombre la respuesta efectiva a los cambios del contenido de calor corporal depende de factores conductuales y autonómicos, tales como cambios en el tono vascular en la piel y el músculo, la termogénesis generada por temblor y la sudoración. La "temperatura objetivo" puede definirse como

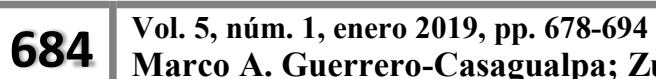


un estrecho rango de temperatura $\left(36.7-37.1{ }^{\circ} \mathrm{C}\right.$ para el hombre) en el cual no se detecta ninguna respuesta efectora. Este punto de corte ha sido denominado también "zona de termoneutralidad" o "intervalo interumbral" y es característico de los humanos. Es de $0.4{ }^{\circ} \mathrm{C}$, pero puede aumentar a $4^{\circ} \mathrm{C}$ durante la anestesia general.

Podemos diferenciar dos compartimentos térmicos en el cuerpo humano, un compartimiento central y uno periférico. El compartimiento central está formado por tejidos con elevada perfusión sanguínea y que mantienen una temperatura relativamente constante. En este compartimento la distribución del calor se realiza a una velocidad mayor que la de los cambios del contenido de calor habituales en el organismo. El compartimento periférico se compone anatómicamente por los miembros superiores e inferiores. Los tejidos que lo forman se caracterizan, en contraposición al compartimento central, por la ausencia de homogeneidad y la variabilidad en el tiempo de la temperatura, la que se encuentra generalmente entre 2 y $4{ }^{\circ} \mathrm{C}$ por debajo de la temperatura central en condiciones ambientales de temperaturas moderadas.

Todo o parte del calor generado en el metabolismo corporal debe ser disipado al ambiente para mantener el estado de normotermia. El 95\% se elimina por la superficie cutánea y una proporción pequeña, vía respiratoria. El 10\% de las pérdidas cutáneas de calor en el adulto se producen por evaporación (en ausencia de sudoración) y ese porcentaje es mayor en niños (especialmente en los pretérminos).

\section{Efectos de la anestesia general}

La anestesia general modifica el intervalo interumbral normal (llevándolo de $0.2{ }^{\circ} \mathrm{C}$ a $4{ }^{\circ} \mathrm{C}$ ) de modo que la respuesta termorregulador compensatoria al frío se desencadena a $\operatorname{los} 34-35^{\circ} \mathrm{C}$ y la respuesta al calor a los $38^{\circ} \mathrm{C}$. La mayoría de los pacientes sometidos a anestesia general desarrollan hipotermia de modo que la temperatura desciende de 1 a $3{ }^{\circ} \mathrm{C}$, dependiendo del tipo de anestesia y su duración, de la magnitud de la exposición quirúrgica y de la temperatura ambiente. La hipotermia intraoperatoria se desarrolla siguiendo un patrón característico. Durante la primera hora de la intervención la temperatura central disminuye de 1 a $1.5{ }^{\circ} \mathrm{C}$. Después de esta fase inicial experimenta una reducción lenta y lineal que dura aproximadamente dos a tres horas y, por último, el paciente entra en una fase de meseta o plateau durante la cual la temperatura permanece constante (Baptista, Rando, \& Zunini, 2010)

\footnotetext{
685 Vol. 5, núm. 1, enero 2019, pp. 678-694 


\section{Anestesia Regional}

La anestesia subaracnoidea inhibe el control central termorregulador a través del bloqueo del sistema nervioso simpático periférico y de los nervios motores, que suprimen la vasoconstricción y los temblores 55-57. La redistribución ocurre en los miembros inferiores y continúa siendo la principal causa de hipotermia perioperatoria en estos pacientes. Su magnitud varía conforme el estado térmico inicial del paciente y puede ser atenuada por el calentamiento de los miembros inferiores antes del inicio de la anestesia. Como hay menor redistribución inicial en pacientes con anestesia regional, la fase de hipotermia lineal será desencadenada a una temperatura más alta, resultando en disminución de la temperatura 50\% menor que con anestesia general. La fase de hipotermia lineal se desarrolla con menor velocidad, una vez que la tasa de producción metabólica de calor permanece próxima a lo normal.

La presencia y la extensión de los bloqueos simpático y motor impide que aparezca la vasoconstricción termorreguladora y esta fase lineal no es interrumpida como ocurre durante la anestesia general. Consecuentemente, pacientes sometidos a intervenciones quirúrgicas de gran porte con anestesia regional corren un riesgo mayor de desarrollar hipotermia grave. Las regiones que no están bajo bloqueo simpático y motor pueden desencadenar respuestas termorreguladoras si el umbral reducido de los temblores es alcanzado, y el paciente no es anciano o no está excesivamente sedado. Entretanto, temblores restrictos a las extremidades superiores del organismo son relativamente ineficaces e insuficientes para la prevención de hipotermia adicional. Hipotermia derivada de anestesia regional es frecuente y depende más de la magnitud y de la duración del procedimiento quirúrgico que de las características del paciente. Raramente la temperatura es medida y en general no se hace diagnóstico de hipotermia durante bloqueos centrales, excepto en los casos en que se espera.

Entre los diferentes locales de monitorización durante anestesia espinal, se constató que medidas más precisas son obtenidas con la temperatura rectal debido a la vasoconstricción cutánea compensatoria arriba del nivel del bloqueo. 


\section{Anestesia Combinada}

Representa la situación de mayor riesgo de hipotermia perioperatoria no intencional. La redistribución inicial en las cuatro extremidades lleva rápidamente a la hipotermia y la fase lineal se desarrolla con mayor velocidad. La anestesia regional disminuye el umbral de vasoconstricción y cuando se suma a la anestesia general tiene su efecto aumentado. Como resultado, la vasoconstricción se desencadena más tardíamente y con menor temperatura. Por otro lado, la anestesia general inhibe los temblores que podrían aumentar la producción interna de calor durante la anestesia espinal. Pero el factor más importante como consecuencia de esta asociación es la eliminación de la vasoconstricción en las extremidades inferiores debido al bloqueo. La vasoconstricción exclusivamente central es ineficaz y la temperatura continúa a declinar, sin alcanzar la fase de meseta. (Biazzotto, Brudniewski, Schmidt, \& Costa, 2006)

\section{Complicaciones de la hipotermia}

La hipotermia conlleva el incremento de la morbilidad, al alterar diversos sistemas y funciones del organismo:

- Escalofríos y activación simpática: Habitualmente los pacientes suelen comentar que los escalofríos son una de las experiencias más desagradables del postoperatorio inmediato, y muchos refieren que su aparición aumenta el dolor durante dicho período.

Los escalofríos se producen como respuesta al frío y son el resultado de una actividad muscular involuntaria que aumenta la producción de calor metabólico. Consisten en contracciones musculares seguidas de relajación a razón de 4-8 ciclos/min. Se desencadenan por señales procedentes de la región prequiasmática del hipotálamo, esta señal se transmite hasta las motoneuronas medulares. Éstas son reclutadas en secuencias, según su tamaño, empezando las motoneuronas seguidas por las pequeñas motoneuronas tónicas a y finalmente por las de mayor tamaño fásicas a. El incremento del metabolismo muscular local puede llegar a un 600\% de forma puntual, pero lo habitual es que si persisten origine un aumento del 100\% del calor basal. Hay que tener presente que los incrementos en el consumo de $\mathrm{O} 2$ pueden constituir un factor de desequilibrio en ciertos pacientes afectos de patologías con gasto cardíaco fijo, shunts intrapulmonares y/o reserva respiratoria limitada. Por otra

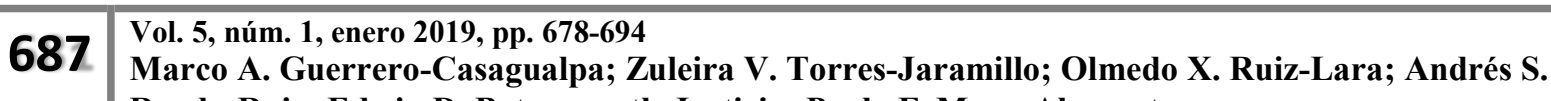
Rueda-Ruiz; Edwin D. Betancourth-Justicia; Paola F. Mena-Alencastro
} 
parte, la hipoxemia suele reducir la aparición y amplitud de los escalofríos. (Campo \& Zaballos, 2003)

\section{Prevención y tratamiento de la hipotermia intraoperatoria no intencionada}

El objetivo de la preservación del calor corporal en los pacientes durante la anestesia y la cirugía consiste en minimizar las pérdidas de calor reduciendo la radiación y convección desde la piel, la evaporación desde las superficies quirúrgicas expuestas y el enfriamiento inducido tras la administración de fluidos fríos intravenosos.

La vasoconstricción termorreguladora intraoperatoria y la termogénesis sin escalofríos (en lactantes) son muy eficaces una vez iniciadas para evitar la hipotermia central. Pero en la mayoría de los pacientes anestesiados, la Temperatura ambiente (Ta) intraoperatoria no desciende lo suficiente como para desencadenarlas. Por este motivo, la hipotermia intraoperatoria no debe contemplarse como una consecuencia inevitable de la cirugía y de la anestesia pues se puede reducir su incidencia al mínimo mediante cualquier técnica que limite la pérdida de calor cutáneo hacia el entorno o que aporte calor al paciente.

Existen diferentes medidas que ayudan a prevenir la aparición de la hipotermia en el período intraperatorio. Prevención de la hipotermia, disminución de las perdidas por redistribución El descenso de la Ta corporal central provocado por la inducción anestésica se debe a la redistribución de calor entre los compartimentos térmicos corporales.

Los únicos métodos que pueden restringir o prevenir las pérdidas de calor por redistribución son la vasodilatación farmacológica preoperatoria y la disminución del gradiente de Ta central-periférico mediante el calentamiento del compartimento periférico y de esta forma reducir el diferencial con el compartimento central. Estas son las únicas técnicas que han resultado útiles para reducir la hipotermia intraoperatoria en cirugías de menos de una hora de duración.

\section{Vasodilatación farmacológica preoperatoria}

La administración de fármacos con efecto vasodilatador antes de la inducción anestésica facilita la transferencia de calor desde el compartimento térmico central al periférico, disminuyendo el gradiente de Ta reduciendo el descenso de la Ta central, pues se mantiene la termorregulación 
fisiológica. Por lo tanto, en el momento de la inducción anestésica, la hipotermia por redistribución es mínima al no existir gradiente de Ta para el flujo térmico central-periférico. Pero estas técnicas preventivas resultan poco útiles en la práctica clínica, pues también hay que tener en cuenta los posibles efectos secundarios no deseados de los fármacos vasodilatadores.

\section{Precalentamiento cutáneo.}

El calentamiento de los tejidos periféricos reduce la hipotermia de redistribución mediante dos mecanismos:

1) disminuyendo el gradiente de Ta central-periférico normal, aumentando la Ta tisular periférica; $\mathrm{y}$

2) induciendo vasodilatación. Por consiguiente, en este caso la inducción anestésica ejerce un escaso efecto vasomotor.

Habitualmente los pacientes hospitalizados están poco abrigados y llegan al quirófano con un considerable gradiente de Ta central-periférico. El calentarlos activamente antes de la inducción anestésica es una de las maneras de conseguir que este gradiente sea mínimo. La eficacia del precalentamiento depende del aumento de la Ta tisular y del contenido calórico del compartimento térmico periférico. Está limitado por el inicio de la producción de sudor, lo que facilita la pérdida de calor, y por la incomodidad provocada por la elevación de la Ta cutánea, lo que obligará a detener el precalentamiento. Esta técnica puede incorporarse sin grandes problemas a la práctica clínica. Debe iniciarse en el momento en el que los pacientes llegan a la zona de espera prequirúrgica y mantenerse durante la monitorización hasta la inducción anestésica. De esta manera, además de evitar las consecuencias de la hipotermia, la vasodilatación facilita las canulaciones venosas y se mantiene a los pacientes cómodos, sin la sensación desagradable de frío que se produce habitualmente al entrar en el quirófano y colocar a los pacientes en la mesa quirúrgica. (Zaballos \& Campos, 2003)

Medidas pasivas para disminuir las pérdidas de calor corporal. Actuación sobre la temperatura ambiente

La Ta ambiente es la variable que más influye para mantener la normotermia en el individuo. Las pérdidas por radiación dependen de la diferencia de Ta entre la del paciente y la del entorno. Todos 
los pacientes anestesiados presentarán hipotermia si la Ta del quirófano es inferior a $21{ }^{\circ} \mathrm{C}$, un $30 \%$ si está entre 21 y $24{ }^{\circ} \mathrm{C}$, y ninguno si es superior a $24^{\circ} \mathrm{C}$. En los quirófanos en los que se realiza cirugía en niños prematuros y recién nacidos 25 la Ta deberá ser de unos $26^{\circ} \mathrm{C}$. Es esencial que cada quirófano disponga de un termostato independiente para ajustar la Ta en cada caso y en cada momento, pues también una Ta ambiente excesiva puede resultar incómoda para el personal que trabaja en el área quirúrgica. Por otro lado, fuera del área quirúrgica (ej.: radiología) hay que tener en cuenta que suele ser imposible ajustar la temperatura ambiente a las necesidades del paciente que va a ser anestesiado. (Zaballos \& Campos, 2003)

\section{Aislamiento pasivo: cubrir la superficie corporal expuesta.}

Aproximadamente el 90\% del calor metabólico se pierde a través de la superficie cutánea, por lo tanto, los sistemas de calentamiento corporal deben proteger al organismo de las pérdidas cutáneas de calor. El método más sencillo para reducir la pérdida de calor por la piel es aplicar un aislamiento pasivo a la superficie cutánea fuera del campo quirúrgico, utilizando los paños quirúrgicos o mantas, pues así se limitan las pérdidas por radiación y convección. La mayor parte del aislamiento térmico la proporciona la piel y el aire inmóvil que queda bajo la cobertura, sin embargo, el acúmulo de capas no incrementa linealmente la protección, por esta razón el añadir más capas no consigue una gran diferencia1. Es más importante el área total de superficie cutánea cubierta que la parte del cuerpo que se proteja o el tipo de material utilizado. Los paños quirúrgicos o una sábana de plástico retienen el calor corporal igual que una manta de algodón (caliente o no) o que las coberturas de plástico metalizadas. En los niños, es muy importante cubrir adecuadamente la cabeza y las extremidades, pues en ellos suponen un gran porcentaje de la superficie corporal total.

La utilización de coberturas de plástico metalizado parece tener poca utilidad en la prevención de la pérdida intraoperatoria de calor. Pero con sólo estas medidas (el mejor aislamiento pasivo raramente reduce la pérdida de calor un 50\%), resulta difícil mantener la normotermia durante toda una intervención quirúrgica, pues sólo se consigue reducir las pérdidas cutáneas y no las asociadas a la relativamente baja Ta ambiente del quirófano o las que conlleva la cirugía. Por este motivo, son necesarios los sistemas de calentamiento corporal activo que mantienen la normotermia mejor que el aislamiento pasivo. 
Sistemas para el calentamiento corporal activo.

La eficacia de los sistemas de calentamiento depende de varios factores entre los que están el diseño del aparato, el tipo de transferencia de calor, su colocación sobre el paciente y, lo que es más importante, el área corporal total con la que se realiza el intercambio de calor. El límite, en cada caso, viene impuesto por la potencia eléctrica de cada uno y por la Ta máxima que pueden tolerar los tejidos corporales sin que se produzcan quemaduras. El riesgo de lesión tisular se incrementa cuando además se asocia la presencia de irritantes químicos como las soluciones desinfectante con iodo, o si la piel es fina y delicada, como en los ancianos.

Por lo tanto, con todos los sistemas de calentamiento cutáneo, para mantener la eficacia y la seguridad se debe calentar tanta superficie cutánea como sea posible, para permitir la máxima transferencia de calor sin que se caliente en exceso ninguna zona.

Para el calentamiento activo se utilizan diversos sistemas que se detallan a continuación:

- Lámparas de infrarrojos

- Colchonetas o mantas por las que circula agua Caliente.

- Aire caliente convectivo

- Mantas eléctricas

- Calentamiento de los fluidos administrados por vía intravenosa

- Calentamiento y humidificación de los gases anestésicos

- Calentamiento de los líquidos de irrigación de cavidades corporales

- Calentadores de CO2 para cirugía laparoscópica (Zaballos \& Campos, 2003)

Otras medidas: administración intravenosa de soluciones de aminoácidos

La administración de aminoácidos por vía intravenosa estimula el gasto energético y la producción y acumulación de calor. Se ha demostrado que durante la anestesia general se incrementa hasta cinco veces el efecto térmico de los aminoácidos, observándose una disminución del temblor postoperatorio. La perfusión perioperatoria de soluciones intravenosas con aminoácidos previene la hipotermia intraoperatoria que se produce habitualmente durante la anestesia general, al aumentar la acumulación de calor y retrasar la estimulación de la producción de calor, sin asociarse a un incremento de la actividad simpática (aumento de la liberación de catecolaminas). A pesar de todo,

691 Vol. 5, núm. 1, enero 2019, pp. 678-694

Marco A. Guerrero-Casagualpa; Zuleira V. Torres-Jaramillo; Olmedo X. Ruiz-Lara; Andrés S.

Rueda-Ruiz; Edwin D. Betancourth-Justicia; Paola F. Mena-Alencastro 
esta técnica no consigue disminuir la pérdida de calor asociada a la inducción anestésica. Sahin et al. (2002) han comprobado además que la técnica anestésica puede influir en el efecto térmico de los aminoácidos, pues observa ron un mayor efecto termogénico cuando se administra ron soluciones con aminoácidos y se utilizó propofol que cuando el anestésico utilizado fue isoflurano. Recientemente, Widman et al (2002) han comprobado que el efecto termogénico de los aminoácidos ocurre también durante la anestesia intradural, aunque en menor medida que con la genera, asociándose además un menor descenso de la temperatura corporal e inferiores pérdidas sanguíneas durante la cirugía. (Zaballos \& Campos, 2003)

\section{Conclusiones.}

La hipotermia perioperatoria es una complicación frecuente y habitualmente subestimada en el paciente quirúrgico y que determina un aumento significativo de efectos adversos como eventos cardiovasculares, infección de la herida quirúrgica así como sangrado intraoperatorio por trastornos en la coagulación. Esto contribuye al aumento de la estadía en sala de recuperación posoperatoria y hospitalaria. El mayor uso de recursos (antibióticos, día de internación, etcétera) seguramente conlleva un aumento de los costos sanitarios. La monitorización de la temperatura durante la cirugía y el mantenimiento de la normotermia son funciones prioritarias e importantes del anestesiólogo. (Baptista, Rando, \& Zunini, 2010)

Numerosos estudios controlados y randomizados demostraron que la hipotermia perioperatoria aumenta la incidencia de efectos adversos: hospitalización prolongada, aumento de la incidencia de infección de la herida operatoria, deterioro de la coagulación y de la inmunidad, isquemia miocárdica, arritmias cardíacas, mayor disconfort del paciente y tiempos de estadía en Sala de Recuperación Posanestésica (SRPA) más prolongados. (Baptista, Rando, \& Zunini, 2010)

Podemos concluir que el calentamiento de la superficie corporal del paciente antes de la inducción anestésica es útil para minimizar el descenso de la temperatura corporal que ocurre tras la inducción, y que el aislamiento pasivo reduce la pérdida de calor corporal. Sin embargo, la mayoría de los pacientes quirúrgicos precisarán de medidas de calentamiento activo para mantenerlos normotérmicos durante el transcurso de la cirugía. Los sistemas más eficaces utilizan aire caliente convectivo o circulación de agua caliente, en diferentes dispositivos pensados para calentar la

\footnotetext{
692 Vol. 5, núm. 1, enero 2019, pp. 678-694 Marco A. Guerrero-Casagualpa; Zuleira V. Torres-Jaramillo; Olmedo X. Ruiz-Lara; Andrés S. Rueda-Ruiz; Edwin D. Betancourth-Justicia; Paola F. Mena-Alencastro
} 
superficie corporal. El calentamiento de los líquidos que se administran por vía intravenosa debe utilizarse cuando se prevea que es necesario perfundir rápidamente cantidades elevadas de los mismos, y debe considerarse sólo como un sistema complementario al calentamiento corporal para mantener la normotermia intraoperatoria (Zaballos \& Campos, 2003)

La literatura coincide que el método más efectivo para contrarrestar la hipotermia es mantener el calentamiento corporal antes de comenzar la cirugía y monitorear durante la misma la temperatura ambiental y del paciente para actuar de manera eficaz en caso de presentar descenso que influya en el riesgo del paciente, en el Ecuador existen pocos estudios que muestren la incidencia de ésta afección de manera general o especifica respecto a la cirugía, edad o patología por tanto los datos estadísticos disponibles no proporcionan una referencia importante como para proyectar y analizar un comportamiento ya sea por el manejo de protocolos médicos, o por alguna predisposición poblacional.

\section{Bibliografía.}

Baptista, W., Rando, K., \& Zunini, G. (2010). Hipotermia perioperatoria. Anestesia Analgesia Reanimación, 24-38.

Biazzotto, C., Brudniewski, M., Schmidt, A., \& Costa, J. (2006). Hipotermia no Período Perioperatorio. Revista Brasilera de anestesiología; 56 (1), 56-66.

Campo, J., \& Zaballos, J. (2003). Hipotermia intraoperatoria no terapéutica: causas, complicaciones, prevención y tratamiento (I parte). Revista Española de Anesteciología y Reanimación; 50, 135-144.

García, L., Cabello, R., Lever, C., Rosales, E., Padilla, R., Garduño, P., \& Gómez, M. (2005). Conceptos actuales en cirugía abdominal de control de daños. Comunicación acerca de donde hacer menos es hacer mas. Trauma; 8 (3), 76-81.

Gómez, M., Ramos, A., Llanes, O., \& Gonzalez, J. (2007). La Tríada de la Muerte”. Acidosis, hipotermia y coagulopatías en pacientes con traumas. Revista Médica Electrónica; 29 (1), 56-64.

Guirao, J., Olmedo, Angela, \& Esperanza, F. (2008). El artículo de revisión. Revista Iberoamericana de Enfermería Comunitaria, http://revista.enfermeriacomunitaria.org/articuloCompleto.php?ID=7.

Hernandez, A., Vinent, J., Delgado, R., \& Castillo, J. (2014). Factores que influyen en la mortalidad en los pacientes graves con cirugia abdominal. Revista Cubana de Cirugía;53(2), 134-144.

\footnotetext{
693 Vol. 5, núm. 1, enero 2019, pp. 678-694

Marco A. Guerrero-Casagualpa; Zuleira V. Torres-Jaramillo; Olmedo X. Ruiz-Lara; Andrés S.

Rueda-Ruiz; Edwin D. Betancourth-Justicia; Paola F. Mena-Alencastro
} 
Tavares, L., Andrade, P., Goné, A., \& Sánchez, P. (2008). Abdomen Abierto. Evolución en su manejo. Cirugia y cirujanos; 76, 177-186.

Zaballos, J., \& Campos, J. (2003). Hipotermia intraoperatoria no terapéutica: prevención y tratamiento. Revista Española de Anestesiología y reanimación; 50, 197-208. 